\title{
Effect of Bed Particle Size on Thermal Performance of a Directly-Irradiated Fluidized Bed Gas Heater
}

\author{
Sae Han Park ${ }^{\circ}$, Chae Eun Yeo, Min Ji Lee and Sung Won Kim * $\mathbb{D}$ \\ School of Chemical and Material Engineering, Korea National University of Transportation, \\ Chungju-si 27469, Chungbuk, Korea; psh4918@ut.ac.kr (S.H.P.); yce@ut.ac.kr (C.E.Y.); mj1226@ut.ac.kr (M.J.L.) \\ * Correspondence: kswcfb@ut.ac.kr; Tel.: +82-43-841-5228
}

\begin{abstract}
There is a growing interest in a fluidized bed particle receiver that directly irradiates sunlight to particles in the fluidized bed as a solar thermal collector for heating. Thermal performance of directly-irradiated fluidized bed gas heater is strongly affected by the physical properties of the particles. The effect of $\mathrm{SiC}$ particle size on heat transfer characteristics in the solar fluidized bed gas heater ( $50 \mathrm{~mm}-\mathrm{ID} \times 100 \mathrm{~mm}$ high) has been determined. The outlet gas temperatures showed a maximum value with increasing gas velocity due to the particles motion by bubble behavior in the bed, and the maximum values were found at 3.6 times of $\mathrm{U}_{\mathrm{mf}}$ for fine $\mathrm{SiC}$ and less than 2.0 times of $\mathrm{U}_{\mathrm{mf}}$ for coarse $\mathrm{SiC}$. Heat absorption from the receiver increased with increasing gas velocity, showing with maximum $18 \mathrm{~W}$ for the fine $\mathrm{SiC}$ and $23 \mathrm{~W}$ for the coarse $\mathrm{SiC}$ at 4.5 times of $\mathrm{U}_{\mathrm{mf}}$. The thermal efficiency of the receiver increased with increasing gas velocity, but was affected by the content of finer particles. The maximum thermal efficiency of the receiver was $14 \%$ for fine $\mathrm{SiC}$ and $20 \%$ for coarse $\mathrm{SiC}$ within the experimental range, but showing higher for the fine $\mathrm{SiC}$ at the same gas velocity. A design consideration was proposed to improve the thermal efficiency of the system.
\end{abstract}

Keywords: solar thermal energy; fluidized bed; gas heater; silicon carbide; particle size

\section{Introduction}

To reduce the environmental impact of huge energy-use in industry, companies continue to devote their efforts to using renewable energy. Among the various renewable energies, solar energy has received the most attention as a promising process application [1]. Solar energy is the most abundant renewable and the cleanest source of energy. Solar energy conversion technology is divided into PV (Solar photovoltaic energy) technology, CSP (Concentrating Solar Power) technology and STC (Solar Thermal Collectors for heating and cooling) technology.

Much progress has been made in PV technology and the CSP technology to reduce carbon dioxide emissions in the power generation sector, which is responsible for a very high fraction of fossil fuel consumption so far. Recently, there is a growing interest in the STC technology in order to produce medium- and low-temperature energy that is applied to processes below $300^{\circ} \mathrm{C}$, such as gas preheating in coal power plant and refinery industries, as well as primary and secondary industries (agriculture, chemical and paper) [2]. The STC technology for utilizing solar energy consists mainly of a solar collection technology for collecting sunlight such as conventional flat plate, parabolic dish and Fresnel lens, and a receiver technology for transferring collected sunlight to an energy carrier. The solar collectors have been continuously developed to improve efficiency; it has recently been found that the performance of a simple flat collector can be greatly improved by optimized configuration with a flat booster bottom reflector [3]. The receiver system can be divided into a direct irradiation method and an indirect irradiation method. The most commonly used method is to indirectly heat the tube for heating the salt or vapor in the tube or to heat the vapor or gas in the transparent tube irradiated 
with sunlight. However, the indirect receiver system may have low heat transfer efficiency due to high resistance in heat transfer. The direct irradiation to water vapor or gas also has a difficulty in efficiently transferring the energy because of the ability of the medium to absorb heat [4].

Thus, there is a growing interest in a fluidized bed particle receiver that directly irradiates sunlight to particles in the fluidized bed, because it has good heat transfer characteristics due to smooth mixing between fluidized particles and gas [5]. Many studies have been conducted to develop the fluidized bed particle receiver and the particles for thermochemical heat storage for the CSP applications [6-8]. However, there have been relatively few studies on the gas heating using this system for the STC application. Although both applications use the principle of fluidized bed, the direct solar heat transfer to energy storage particles is important in the CPS applications, while the heat transfer of absorbed energy in the solid to the gas is also very important [1,4]. Thus, the study on particle behavior as an energy transfer medium in the gas-solid fluidized bed is an important part of the STC field.

The thermal efficiency of the fluidized bed particle receiver is strongly influenced by the physical properties of the particles at a given operating temperature $[9,10]$. It has been found that an optimal particle size is ranged between 500 and $1000 \mu \mathrm{m}$ in mean size diameter for the fluidized bed receiver [7]. However, it is expected that the size depends on the operating temperature [7]. So far, most studies on the effect of particle properties have been carried out in the receiver at high temperature over $600{ }^{\circ} \mathrm{C}$, and the research in the medium and low temperature region below $300{ }^{\circ} \mathrm{C}$ is relatively sparse. [1,7] Moreover, many studies have been conducted using xenon lamps as a heat source in the simulated indoor space, not the outdoor environment $[8,11,12]$.

In this study, thermal performance experiments were carried out in a directly-irradiated fluidized bed of different particles in the outdoor environment for the application of gas heating. The effect of $\mathrm{SiC}$ particle size on the thermal efficiency of the preheater has been determined.

\section{Experimental}

Experiments were carried out in a solar energy particle receiver system as in Figure 1 . The fluidized bed gas heating system has two parts such as a solar collector and a particle receiver. The solar collector system consists of two reflective mirrors (model 1100, $3 \mathrm{M}$ ) with reflectance of 93\%, Fresnel lens (effective ID $=457 \mathrm{~mm}$, Edmund optics) and focal lens. The first reflective mirror was installed in front of the Fresnel lens in order to chase the direct sunlight of the moving sun as in Figure 1a. The Fresnel lens has a light transmittance of 92\%. The solar power meter (Procal1333R, TES) was installed on the Fresnel lens, which faces the first reflective mirror to measure the directly nominal irradiance supplied into the fluidized bed through the Fresnel lens. It was checked periodically whether the light intensity changed in radial direction across the Fresnel lens.

The fluidized bed particle receiver was made of Pyrex glass and has a main column (50 mm-ID $\times 100 \mathrm{~mm}$ high) as shown in Figure 1b. The main column has the enlarged top section (110 mm-ID) to reduce the particles' elutriation. A highly transparent quartz plate is located at the top surface of the column to get high transmittance for light irradiation. The air as a fluidizing-gas was introduced into the column through a distributor via a mass flow meter (RK1150, Kojima instrument, Kyotanabe Kyoto, Japan). A distributor of sintered plate type was used to inject the air. Pressure drops across the bed were measured at two pressure taps, which were located at column wall around the inlet and outlet to get the information of solid holdup. A total of 5 thermocouples (K-type) were installed to measure the temperatures of inlet air, outlet air and bed particles. 


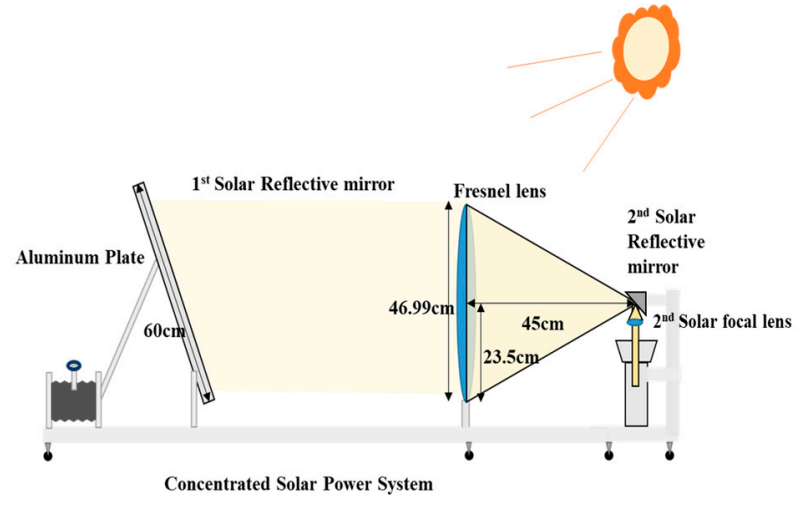

(a)

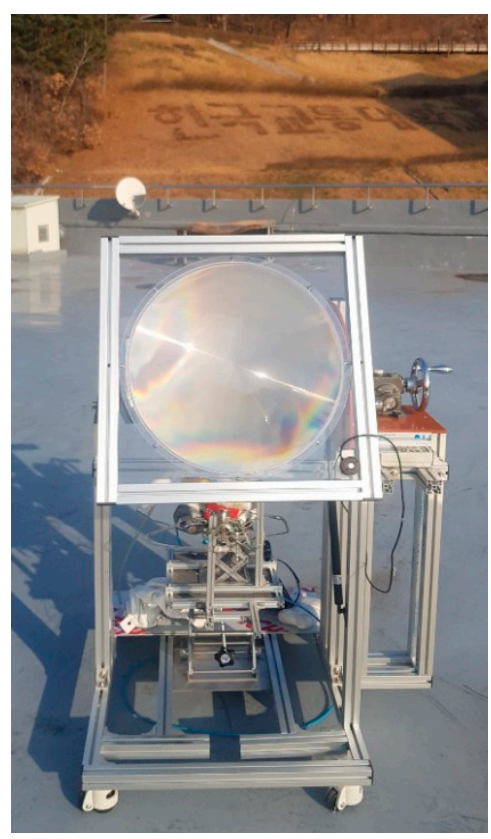

(c)

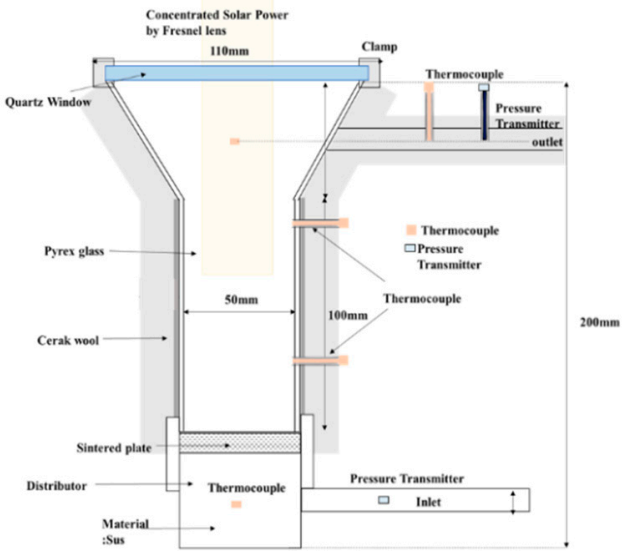

(b)

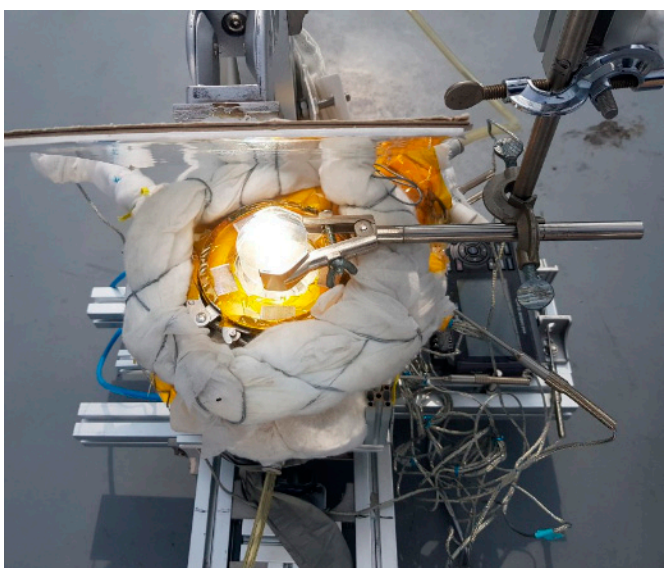

(d)

Figure 1. Experimental apparatus of solar heat gas heater system: (a) solar light collector, (b) particle receiver, (c) photo of the system in the field test, (d) top view of the particle receiver in the field test.

Two kinds of silicon carbide $(\mathrm{SiC})$ particles, a good absorber of the radiation, were adopted as bed materials to investigate the effect of the particle diameter. The physical properties of the SiC particles are shown in Table 1. They have almost the same particle density but different mean particle diameter and size distribution (PSD) based on sieve analysis as shown in Figure 2a. The fine SiC particles (F220, Showa denko, Tokyo, Japan) have the particle mean diameter of $0.052 \mathrm{~mm}$ and the apparent particle density of $2992 \mathrm{~kg} / \mathrm{m}^{3}$, corresponding to the Geldart A group based on Geldart classification [13]. The coarse SiC particles (F100, Showa denko, Tokyo, Japan) have the particle mean diameter of $0.123 \mathrm{~mm}$ and the apparent particle density of $3015 \mathrm{~kg} / \mathrm{m}^{3}$, corresponding to the Geldart B group [13]. 
Table 1. Physical properties of SiC (Silicon carbide) particles used in the study.

\begin{tabular}{cccc}
\hline Particles & $\begin{array}{c}\text { Average Particle Diameter, } \\
\mathbf{d}_{\mathbf{p}}[\mu \mathrm{m}]^{(\mathbf{a})}\end{array}$ & $\begin{array}{c}\text { Apparent Density, } \boldsymbol{\rho}_{\mathbf{p}} \\
{\left[\mathbf{k g} / \mathbf{m}^{\mathbf{3}}\right]^{(\mathbf{b})}}\end{array}$ & ${\text { Reflectivity }[\%]^{(\mathbf{c})}}^{(2992}$ \\
\hline Fine $\mathrm{SiC}$ & 52 & 3015 & 11.2 \\
\hline Coarse $\mathrm{SiC}$ & 123 & 9.0 \\
\hline
\end{tabular}

(a) based on the sieve analysis; ${ }^{\text {(b) }}$ based on measurement with pycnometer (ASTM D 854-14 [14]); ${ }^{\text {(c) }}$ based on analysis (wave length: 400-2000 nm)) with UV/Vis/NIR Spectrometer (LAMBDA750, PerkinElmer, Waltham, MA, USA).

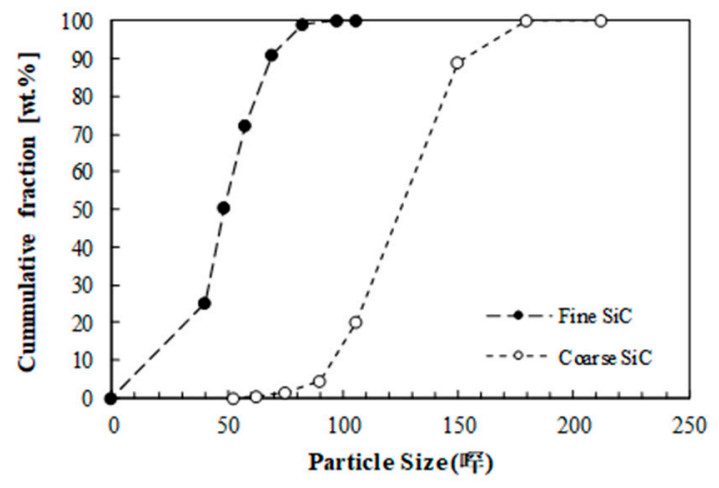

(a)

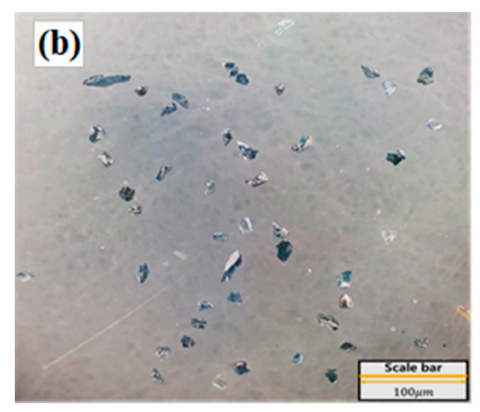

(b)

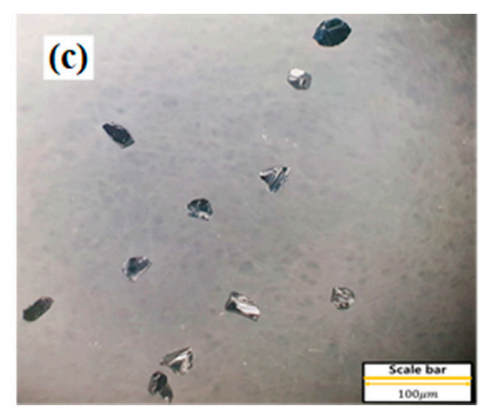

(c)

Figure 2. (a) Particle size distributions of SiC particles, Magnified images of (b) fine SiC particles and (c) coarse $\mathrm{SiC}$ particles.

In comparison of the PSD (Figure 2a), the fine $\mathrm{SiC}$ has $25.2 \mathrm{wt} \%$ of of fine particles less than 45 microns $\left(\mathrm{F}_{45}\right)$, but the coarse $\mathrm{SiC}$ has no $\mathrm{F}_{45}$. The static bed height was $45 \mathrm{~mm}$ with the bed materials of $120 \mathrm{~g}$. The solar irradiation was concentrated by the Fresnel lens, and the light was directed toward the surface of the $\mathrm{SiC}$ particles in the fluidized bed at given gas velocity, as in Figure 1d. The focal area of the light on the particle bed surface is about $1257 \mathrm{~mm}^{2}$. The gas velocity was in the range of $0-0.11 \mathrm{~m} / \mathrm{s}$. The experiment was performed in an outdoor steady state [15]. The air was injected from a compressor and maintained at a constant temperature. Tests were carried out after determining steady state that the air outlet temperature from the particle receiver is constant under the condition where the solar intensity did not change significantly for a certain period.

\section{Results and Discussion}

It is very important to obtain the minimum fluidization velocity $\left(\mathrm{U}_{\mathrm{mf}}\right)$ for determining the optimal operating condition of the particle receiver considering the gas-solid behavior in the fluidized bed [8]. Figure 3 shows variations of the pressure drop across the bed with varying gas velocity for determining the $U_{\mathrm{mf}}$. The pressure drop across the fixed bed increases linearly due to the increase in kinetic energy loss and friction loss with the increasing of the gas velocity through the voidage in the bed. The pressure drop then reaches a constant value that is approximately equal to the weight of the bed material, 
meaning that all of the bed materials are fluidized. As shown in the Figure 3, the $U_{m f}$ is determined from the intersection of two pressure drop change lines with different slopes $[8,16]$. From Figure 3 , the $\mathrm{U}_{\mathrm{mf}}$ of fine $\mathrm{SiC}$ particles is about $0.006 \mathrm{~m} / \mathrm{s}$. The coarse particle $\mathrm{U}_{\mathrm{mf}}$ is $0.017 \mathrm{~m} / \mathrm{s}$, which is about three times as high as that of fine particle $\mathrm{U}_{\mathrm{mf}}$. It is believed that the system with fine $\mathrm{SiC}$ particles is capable of operating in the fluidized bed at a lower gas velocity range. In addition, it is more likely that higher gas temperatures can be achieved compared to the coarse SiC bed due to sufficient fluidized bed-gas contact time at lower gas velocities and larger specific surface area of the particles.

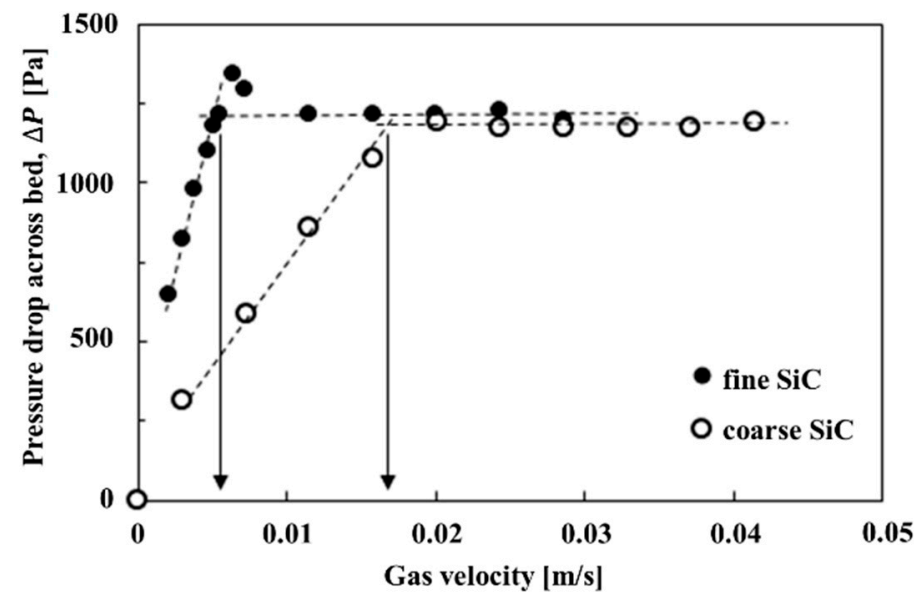

Figure 3. Pressure drop-versus-velocity diagram for determination of $U_{\mathrm{mf}}$.

The performance of the solar energy system depends strongly on the weather, so it is necessary to measure the reachable temperature of the system and to analyze the system feasibility and thermal efficiency based on the results of the temperature variation. The effect of gas velocity on temperatures of bed and outlet gas in the fluidized bed receiver is shown in Figure 4. In this study, the temperature of the bed material was measured at a location of $60 \%$ of the total bed height, which is $2 \mathrm{~cm}$ below the surface exposed to solar light. Fine $\mathrm{SiC}$ particles showed higher temperature than coarse particles. However, the comparison of the results between the particles is not meaningful here, because the obtainable temperature in the receiver depends on the weather condition at the measurement date [17]. Figure 4 shows well the difference in the direct normal irradiance (DNI) values, which is the amount of solar radiation received per unit area, due to the different experiment dates of the two particles. The outlet gas temperature of both particles was lower than the bed temperature at low gas velocity, indicating a heat loss through the light transmission window at the top of the reactor (Figure 1d). However, as the gas velocity increases, the heat absorption amount increases and the residence time of the gas in the freeboard decreases so that the outlet gas temperature approaches the bed temperature. The bed temperature and outlet gas temperature showed maximum values with variation of the gas velocity, because the particle behavior in the fluidized bed is strongly affected by the bubbles' formation and their motion in the bed. More specifically, the formation of gas bubbles in the fluidized bed and the upward flow of the bubbles give flywheel effect in the fluidized bed, giving better mixing and convective heat transfer effects of the gas-particles in the whole fluidized bed [18]. A gulf streaming in the bed with downflow of emulsion at the center is induced by the uneven rising bubbles with the increase in gas velocity $[13,19]$, enhancing the mixing of the hot particles on the bed surface with the other region of the bed and making the temperature distribution in the whole bed uniform. 


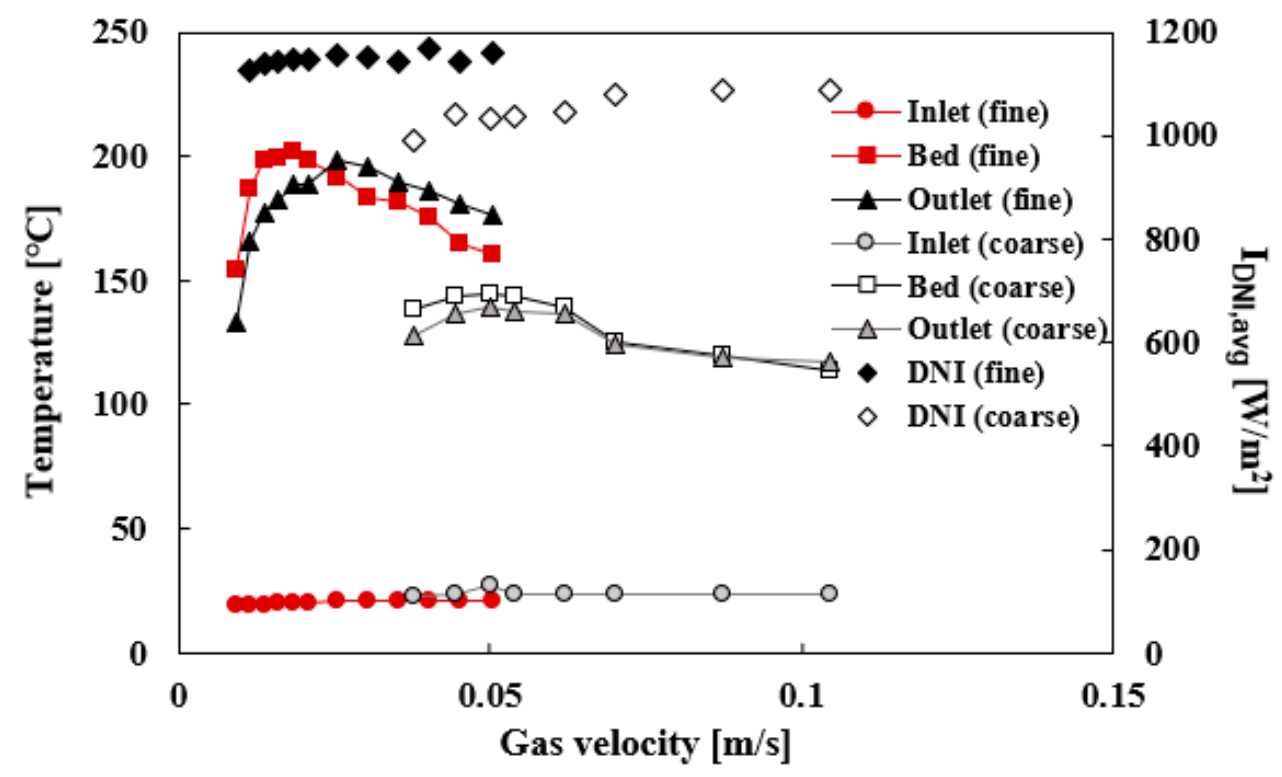

Figure 4. Effect of gas velocity on temperatures of bed and outlet gas in the particle receiver.

The bubble fraction increases with further increasing of the gas velocity, resulting in increased direct heat transfer efficiency of the particles to gas due to the vigorous mixing in the bed. The outlet gas temperature also increases with the increase in heat transfer efficiency. However, the temperature also decreases with the further increase in gas velocity due to an increase in contacting resistance between the gas in enlarged bubble and the particles, and a reduction in the gas residence-time in the bed of the reactor [18]. In the comparison of the particles, the maximum value was found at 3.6 times of $\mathrm{U}_{\mathrm{mf}}$ (3.6 times of minimum fluidization velocity) for fine $\mathrm{SiC}$, and the maximum at less than 2.0 times of $U_{\mathrm{mf}}$ for coarse particles. The maximum value at different $\mathrm{U}_{\mathrm{mf}}$ numbers is due to the different fluidization characteristics between the two particles. Coarse $\mathrm{SiC}$ belonging to Geldart group $B$ showed a maximum temperature at the initial stage due to the immediate generation of bubbles after the $\mathrm{U}_{\mathrm{mf}}$, whereas fine $\mathrm{SiC}$ belonging to the Geldart group A generally produces bubbles at 2-3 times of $U_{\mathrm{mf}}$, resulting in the maximum temperature appearing relatively late [20]. Interestingly, the fine $\mathrm{SiC}$ exhibited a peculiar phenomenon where the outlet gas temperature was higher than the bed temperature, unlike the coarse $\mathrm{SiC}$, with further increase in the gas velocity above $0.023 \mathrm{~m} / \mathrm{s}$. This is because the fine $\mathrm{SiC}$ with high contents of fines $\left(\mathrm{F}_{45}\right.$ : particles less than $45 \mu \mathrm{m}$ [16]) are easily entrained upward at a high gas velocity [16], resulting in further heat transfers to the gas in the expanded column. The coarse SiC with a relatively small amount of fines showed similar bed and outlet gas temperatures as the gas velocity increased.

An analysis in viewpoint of dimensionless temperature with height is important for understanding thermal performance of reactor design. [21]. In order to examine the relation between the particle behavior in the bed and the thermal performance of the reactor, this study introduces a dimensionless temperature variation as

$$
\mathrm{T}^{*}=\left(\mathrm{T}_{\text {bed }}-\mathrm{T}_{\text {inlet }}\right) /\left(\mathrm{T}_{\text {outlet }}-\mathrm{T}_{\text {inlet }}\right)
$$

Figure 5 shows variations of the dimensionless temperature $\left(\mathrm{T}^{*}\right)$ with gas velocity and fluidization number $\left(\mathrm{U}_{\mathrm{g}} / \mathrm{U}_{\mathrm{mf}}\right)$. An ideal fluidized bed heat exchanger has 1.0 of the $\mathrm{T}^{*}$ where bed and outlet temperatures are the same. The dimensionless temperature was higher than 1.0 at low gas velocity and decreased below 1.0 with increasing gas velocity. A value above 1.0 at low gas velocities means that the gas absorbed heat from the bed material as it passed through the fluidized bed, but the temperature of the gas was lowered due to heat loss through the solar-transmission window above the bed surface (Figure 1d). This means that the control of heat loss in the expanded column is more important than that inside the bed to improve thermal performance at low gas velocities. However, the $\mathrm{T}^{*}$ reached 1.0 
at a certain gas velocity, which means that enhanced gas-solid heat transfer occurred over heat loss through the window. Interestingly, the system showed that both particles reached 1.0 at the fluidization number of about 4.5 times of $U_{m f}$, as shown in Figure $5 b$, where the fluidization number corresponds to the velocity as the gas starts to rise homogeneously through the bed [22]. The fine SiC having large amounts of easily entrained fines exhibits a relatively low $\mathrm{T}^{*}$ at a high gas velocity compared to coarse $\mathrm{SiC}$, confirming that the additional heat transfer in the freeboard region has a greater effect on the overall heat transfer. Therefore, it is considered that control of heat-transfer inside the bed, such as bubble size control, becomes more important at high gas velocity.
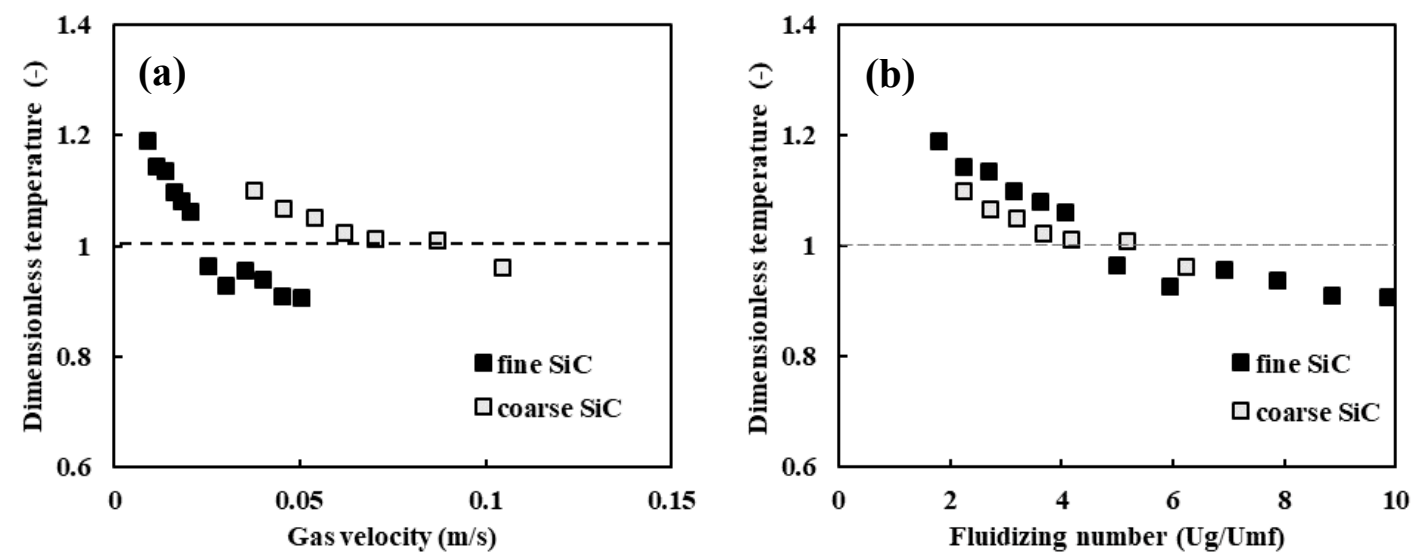

Figure 5. Effects $t$ of (a) gas velocity and (b) fluidizing number on dimensionless temperature $\left(\mathrm{T}^{*}\right)$.

Figure 6 shows an impact of gas velocity on heat absorption by gas in the particle receiver. The heat energy is obtained by Equation (2) as

$$
\mathrm{Q}_{\text {gas }}=\rho_{\text {gas }} \mathrm{U}_{\mathrm{g}} \mathrm{A} \mathrm{C}_{\mathrm{p} \text {,gas }}\left(\mathrm{T}_{\text {outlet }}-\mathrm{T}_{\text {inlet }}\right)
$$

where, $Q_{\text {gas }}$ is heat energy per unit time of the gas, $\rho_{\text {gas }}$ is gas density, $A$ is reactor area, and $C_{p, g a s}$ is the heat capacity of the gas. The heat absorption from the receiver increased as gas velocity increased. As mentioned earlier, the increase in gas velocity increases the mixing of the bed materials in the fluidized bed and the temperature uniformity in the bed due to the increases in bubble frequency [18]. Eventually, this increases the direct gas-solid heat transfer rate, thereby increasing the amount of energy delivered to the gas. The heat transfer rate and thus the exit gas temperature slightly decrease as the bubble fraction increases with further increase in the gas velocity, but the resulting amount of heat absorption increases due to the increased amount of injected air. In this study, maximum $18 \mathrm{~W}$ with fine $\mathrm{SiC}$ and $23 \mathrm{~W}$ with coarse $\mathrm{SiC}$ were obtained within the experimental range. The fine SiC were also expected to obtain higher amounts of heat absorption through additional heat transfer by the entrained fines in the freeboard region as the gas velocity increases, but the increase in the fines entrainment to exit region causes an excessive release of particles, thus limiting the experimental range in the current system. It will be possible to improve this by installing particle recovery systems such as internal cyclones $[13,23]$. On the other hand, coarse $\mathrm{SiC}$ particles without the fines could obtain higher amounts of energy through operation at higher flow rates. 


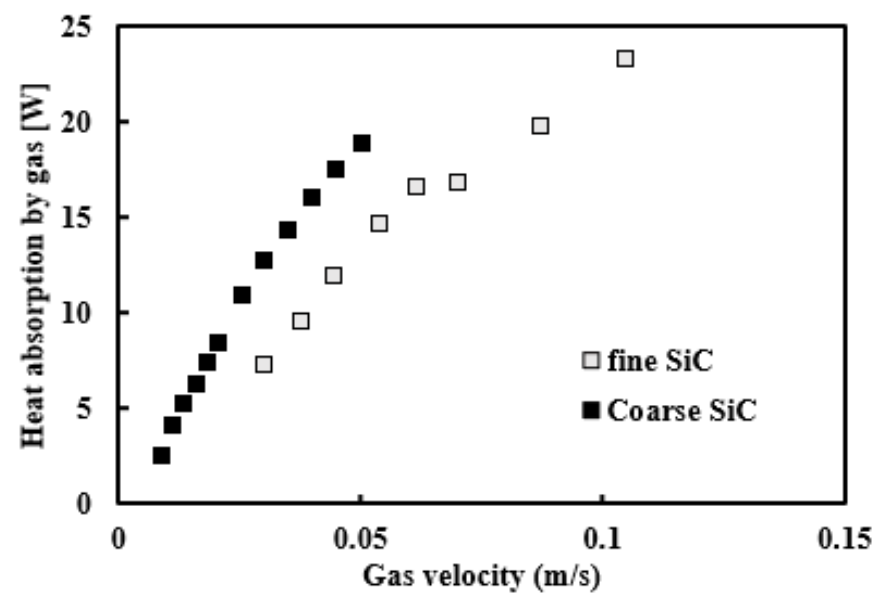

Figure 6. Effect of gas velocity on heat absorption by gas in particle receiver with different SiC.

In order to calibrate the daylight difference or DNI variation between test dates as in Figure 6, the thermal efficiency comparison of the receiver is shown in Figure 7. The thermal efficiency is obtained by Equations (3) and (4) as

$$
\begin{gathered}
\eta_{\text {thermal }}=\mathrm{Q}_{\text {gas }} / \mathrm{Q}_{\text {solar }} \\
\mathrm{Q}_{\text {solar }}=\mathrm{I}_{\mathrm{DNI}} \mathrm{A}_{\text {Fresnel }} \rho_{\text {mirror }} \tau_{1, \text { Fresnel }} \tau_{1, \text { focal }} \tau_{\text {quartz }}
\end{gathered}
$$

where, $\eta_{\text {thermal }}$ is energy efficiency of the system, $Q_{\text {solar }}$ is solar energy per time obtained by the solar collector system, $I_{D N I}$ is intensity of direct normal irradiance, $A_{\text {Fresnel }}$ is Fresnel lens area, $\rho_{\text {mirror }}$ is reflectance of the second reflect mirror, $\tau_{1}$ is transmittance of lens, and $\tau_{\text {quartz }}$ is transmittance of transparent quartz plate. The thermal efficiency increased with an increase in gas velocity due to the increased heat transfer in the bed by the increased bubble fraction. Maximum thermal efficiencies of $14 \%$ for fine $\mathrm{SiC}$ and $20 \%$ for coarse $\mathrm{SiC}$ were obtained within the experimental range. These are relatively low values compared to the previous report, caused by a large heat loss out of the system in the field [24]. In comparison of the two particles, the fine SiC showed higher thermal efficiency than the coarse $\mathrm{SiC}$ when compared at the same gas velocity (dotted box in Figure 7). This is the opposite of what is reported in the high temperature receiver $[9,10]$. In this receiver, the high thermal performance of the fine $\mathrm{SiC}$ is caused by the particle properties and its behavior such as large specific area and small heat transfer resistance due to small bubble, which are characteristics of Geldart A group particles [20]. However, the energy efficiency of the fine $\mathrm{SiC}$ particles did not show a significant increase compared to the coarse $\mathrm{SiC}$, contrary to expectations based on the hydrodynamic behavior of the particles shown in Figures 4 and 5. This unexpected result is due to the difference in fines content $\left(\mathrm{F}_{45}\right)$ of SiC particles. Coarse $\mathrm{SiC}$ has no $\mathrm{F}_{45}$, while fine $\mathrm{SiC}$ has an $\mathrm{F}_{45}$ content of $25.2 \mathrm{wt} \%$. The fines below $45 \mu \mathrm{m}$ in the fluidized bed generally serve as additives to aid fluidization, such as lubricants, between particles in the fluidized bed [16]. However, it was observed that the fines are easily entrained at low gas velocity [25] and attached to the solar light-transmission plate by van der Waals force [26-28] as shown in Figure 8a. This lowers the light transmissivity of the sunlight collected by the Fresnel lens to the receiver, resulting in a lower thermal efficiency of the system. However, the effect of the fines still seems to predominate the positive effect under a certain condition due to the enhanced heat transfer in the freeboard region as shown in a comparative experiment carried out at $0.05 \mathrm{~m} / \mathrm{s}$ shown in Figure $8 \mathrm{~b}$. Therefore, it is desirable to reduce the content of smaller fines, which easily adhere to the transmission window, in order to further improve the system's efficiency. Further study is required to define the smaller fines and to optimize the PSD of the bed considering the effects of the opposing effects of the fines. 


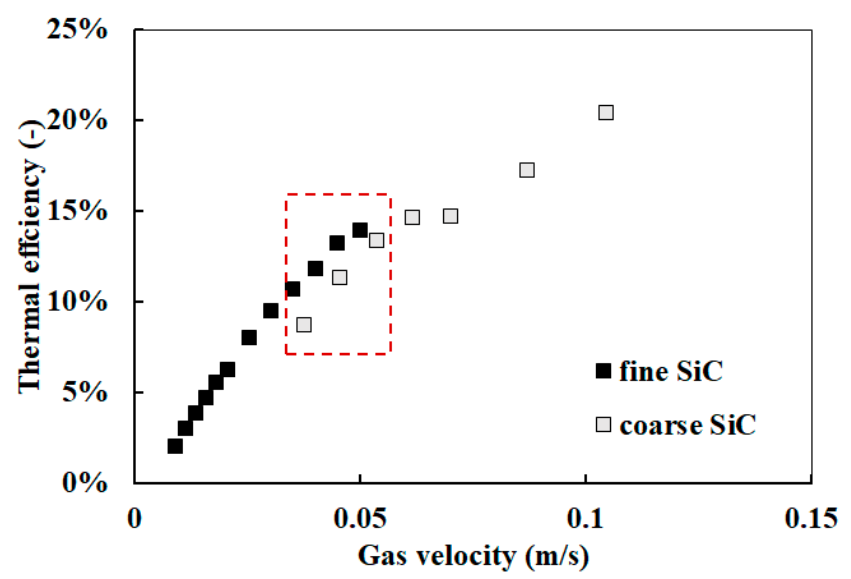

Figure 7. Effect of gas velocity on the thermal efficiency of particle receiver with different SiC.

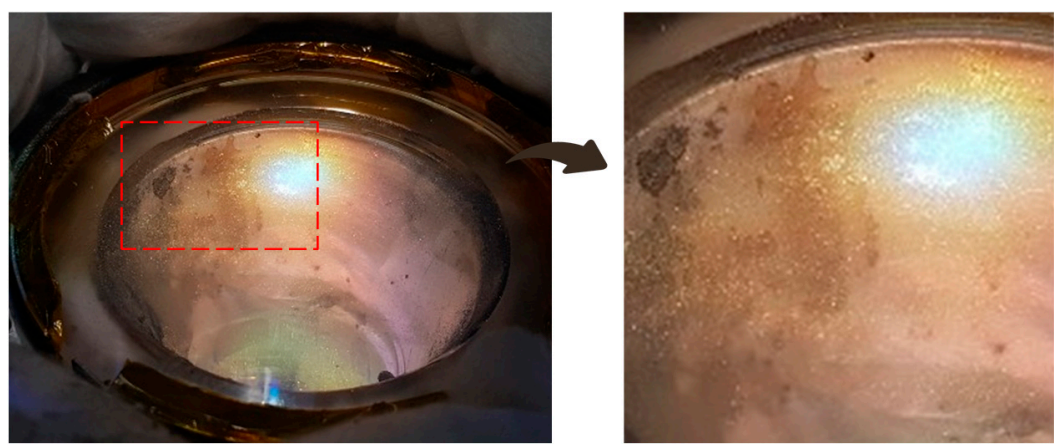

(a)

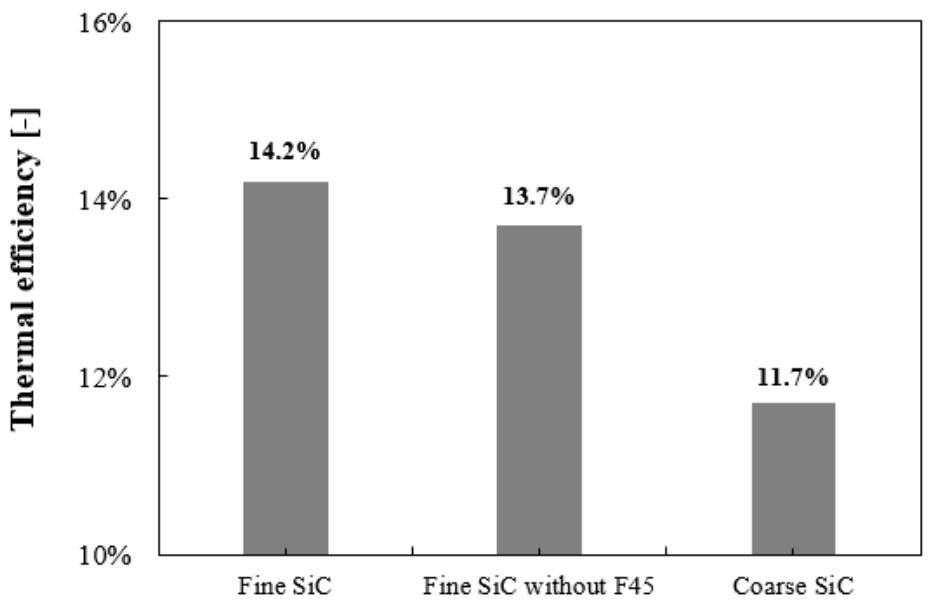

(b)

Figure 8. Effect of fine powder less than $45 \mu \mathrm{m}$ in bed materials on light transmissivity: (a) fine power stuck to window; (b) Effect of fine powder on thermal efficiency at $U_{g}=0.05 \mathrm{~m} / \mathrm{s}$.

The design and performance of the fluidized bed heat exchanger has been optimized depending on particle properties and operating conditions such as gas velocity [29]. The results of this study could thus provide information for further improvement of the solar particle receiver compatible with solar light collecting system. The small particles in the Geldart A group have positive effects such as improved fluidity in the bed and additional heat transfer in the freeboard region in viewpoint of the fluidized bed, but have a negative effect on the light transmittance in viewpoint of the solar system. In addition, the application of an expanded column above the reactor to reduce the amount of particles entrained at high gas velocity has a negative effect of providing heat loss due to the increase in gas residence time in the upper region. Therefore, for further improvement of the system's thermal 
efficiency, further studies on the design of the reactor freeboard section that can reduce the gas residence time as well as the optimum particulate content in the Geldart A particles to minimize the negative effects are required [30]. In addition, an improvement such as a double transmission window will also be required in order to minimize the energy loss through the transmission window in which external insulation is difficult.

\section{Conclusions}

The effect of bed particle size on thermal performance of the directly-irradiated fluidized bed gas heater has been determined. The performance of the system was greatly affected by the physical properties of the particles with an increase in gas velocity. The temperatures of the bed and the outlet gas showed maximum points with gas velocity, and the maximum values were found at 3.6 times of $\mathrm{U}_{\mathrm{mf}}$ for fine $\mathrm{SiC}$ and less than 2.0 times of $\mathrm{U}_{\mathrm{mf}}$ for coarse $\mathrm{SiC}$. Heat absorption and thermal efficiency from the receiver increased with increasing gas velocity, showing with maximum $18 \mathrm{~W}$ and $14 \%$ for the fine $\mathrm{SiC}$ and $23 \mathrm{~W}$ and 20\% for the coarse SiC. Design considerations on the fine content in bed materials and reactor geometry were proposed to improve the thermal efficiency of the system.

Author Contributions: S.H.P. conducted the research, wrote the paper, C.E.Y. and M.J.L. analyzed the data, S.W.K. reviewed, edited and supervised. All authors have read and agreed to the published version of the manuscript.

Funding: This research was funded by the Ministry of Education (NRF-2019R1A2C1011671).

Acknowledgments: This research was supported by Basic Science Research Program through the National Research Foundation of Korea (NRF) funded by the Ministry of Education (NRF-2019R1A2C1011671).

Conflicts of Interest: The authors declare no conflict of interest.

\section{References}

1. Wang, F.; Bai, F.; Wang, T.; Li, Q.; Wang, Z. Experimental study of a single quartz tube solid particle air receiver. Sol. Energy 2016, 123, 185-205.

2. Jia, T.; Huang, J.; Li, R.; He, P.; Dai, Y. Status and prospect of solar heat for industrial processes in China. Renew. Sustain. Energy Rev. 2019, 90, 475-489.

3. Roberto, B.; Frattolillo, A.; Mastino, C.; Curreli, S.; Ghiani, E. A comprehensive optimization model for flat solar collector coupled with a flat booster bottom reflector based on an exact finite length simulation model. Energy Convers. Manag. 2018, 164, 482-507.

4. Li, S.; Kong, W.; Zhang, H.; Sabatier, F.; Ansart, R.; Flamant, G.; Baeyens, J. The fluidized bed air heater exchanger in a hybrid Brayton-cycle solar power plant. In Proceedings of the 6th ASCON-IEEChE, Taiwan, China, 4 November 2018.

5. Alonso, E.; Romero, M. Review of experimental investigation on directly irradiated particles solar reactors. Renew. Sustain. Energy Rev. 2015, 41, 53-67.

6. Gokon, N.; Ono, R.; Hatamachi, T.; Liuyun, L.; Kim, H.; Kodama, T. $\mathrm{CO}_{2}$ gasification of coal cokes using internally circulating fluidized bed reactor by concentrated Xe-light irradiation for solar gasification. Int. J. Hydrogen Energy 2012, 37, 12128-12137. [CrossRef]

7. Flamant, G. Theoretical and experimental-study of radiant-heat transfer in a solar fluidized-bed receiver. AIChE J. 1982, 28, 529-535. [CrossRef]

8. Matsubara, K.; Kazuma, Y.; Sakurai, A.; Suzuki, S.; Lee, S.J.; Kodama, T.; Gokon, N.; Choi, H.S.; Yoshida, K. High-temperature Fluidized Receiver for Concentrated Solar Radiation by a Beam-down Reflector System. Energy Procedia. 2014, 49, 447-456. [CrossRef]

9. Ma, Z.; Mehos, M.; Glatzmaier, G.; Sakadjian, B.B. Development of a concentrating solar power system using fluidized-bed technology for thermal energy conversion and solid particles for thermal energy storage. Energy Procedia 2015, 69, 1349-1359.

10. Ho, C.K. A review of high-temperature particle receivers for concentrating solar power. Appl. Therm. Eng. 2016, 109, 958-969. [CrossRef]

11. Briongos, J.V.; Gomez-Hernandez, J.; Gonzalez-Gomez, P.A.; Serrano, D. Two-phase heat transfer model of a beam-down gas-solid fluidized bed solar particle receiver. Sol. Energy 2018, 171, 740-750. [CrossRef] 
12. Tregambi, C.; Chirone, R.; Montagnaro, F.; Salatino, P.; Solimene, R. Heat transfer in directly irradiated fluidized beds. Sol. Energy 2016, 129, 85-100. [CrossRef]

13. Kunii, D.; Levenspiel, O. Fluidization Engineering, 2nd ed.; Butterworth-Heinemann: Waltham, MA, USA, 1991.

14. ASTM D854-14. Standard Test Methods for Specific Gravity of Soil Solids by Water Pycnometer; ASTM International: West Conshohocken, PA, USA, 2014.

15. Roberto, B.; Ubaldo, C.; Setfano, M.; Roberto, I.; Elisa, S.; Paolo, M. Graybox and adaptative dynamic neural network identification models to infer the steady state efficiency of solar thermal collectors starting from the transient condition. Sol. Energy 2010, 84, 1027-1046.

16. Kim, S.W.; Yeo, C.E.; Lee, D.Y. Effect of Fines Content on Fluidity of FCC Catalysts for Stable Operation of Fluid Catalytic Cracking Unit. Energies 2019, 12, 293.

17. Zhang, Y.N.; Bai, F.; Zhang, X.; Wang, F.; Wang, Z. Experimental study of a single quartz tube solid particle air receiver. Energy Procedia 2015, 69, 600-607. [CrossRef]

18. Kim, S.W.; Kim, S.D. Heat transfer characteristics in a pressurized fluidized bed of fine particles with immersed horizontal tube bundle. Int. J. Heat Mass Transf. 2013, 64, 269-277.

19. Kim, S.W.; Kim, S.D. Void properties in dense bed of cold-flow fluid catalytic cracking regenerator. Processes 2018, 6, 80. [CrossRef]

20. Abrahamsen, A.R.; Geldart, D. Behaviour of Gas-Fluidized Beds of Fine Powders Part I. Homogeneous Expansion. Powder Technol. 1980, 26, 35-46.

21. Chinsuwan, A.; Dutta, A. Investigation of the temperature distribution and material limits of membrane water wall tubes of circulating fluidized bed boilers. Can. J. Chem. Eng. 2010, 88, 144-152.

22. Cranfield, R.R.; Gliddon, B.J. Absorption in Shallow Fluidized Beds of Large Particles. Inst. Chem. Engrs. Symp. Series 1976, 38, H4.

23. Yang, W. Handbook of Fluidization and Fluid-Particle Systems; Marcel Deckker Inc: New York, NY, USA, 2003; pp. 1-27.

24. Gomez-Garcia, F.; Gauthier, D.; Flamant, G. Design and performance of a multistage fluidised bed heat exchanger for particle-receiver solar power plants with storage. Appl. Energy 2017, 190, 510-523.

25. Yeo, C.E.; Kim, S.W. Axial holdup distributions of FCC catalysts in a small-diameter riser with abrupt exit in circulating fluidized bed. Adv. Powder Technol. 2020, 31, 1946-1956.

26. Kim, S.W. Effect of height on CNT aggregates size and shape in freeboard region of a fluidized bed. Korean Chem. Eng. Res. 2019, 57, 105-110.

27. Zhu, C.; Yu, Q.; Dave, R.N. Gas fluidization characteristics of nanoparticle agglomerates. AIChE J. 2005, 51, 426-439. [CrossRef]

28. Cocco, R.; Issangya, A.; Karri, S.B.R.; Freeman, T.; Jaeger, H.M.; Knowlton, T.M. Small-Scale Particle Interactions Are Having Significant Effects on Global Fluidized Bed Behavior. KONA Powder Part. J. 2017, 34, 155-167.

29. Chen, J.C.; Grace, J.R.; Golriz, M.R. Heat transfer in fluidized beds: Design methods. Powder Technol. 2005, 150, 123-132. [CrossRef]

30. Ho, C.K. Advances in central receivers for concentrating solar applications. Sol. Energy 2017, 152, 38-56. [CrossRef]

(C) 2020 by the authors. Licensee MDPI, Basel, Switzerland. This article is an open access article distributed under the terms and conditions of the Creative Commons Attribution (CC BY) license (http://creativecommons.org/licenses/by/4.0/). 